\title{
Railway track layout modelling and its application to an automatic route setting system
}

\author{
H. Teshima ${ }^{1}$, S. Hori ${ }^{1}$, A. Shimura ${ }^{1} \&$ N. Sato ${ }^{2}$ \\ ${ }^{I}$ Yokohama Research Laboratory, Hitachi, Ltd., Japan \\ ${ }^{2}$ Infrastracture Systems Company, Hitachi, Ltd., Japan
}

\begin{abstract}
Automatic Route Setting (ARS) is a major subsystem of railway traffic management systems. ARS automatically sets routes in accordance with timetables, train descriptions and facility situations. To develop the ARS system, various conditions have to be considered, such as train operations, track layouts, and equipped facilities. This leads to a huge amount of work in ARS development aimed at dealing with software variations among ARS products. In the software development area, model-based development is commonly used to improve productivity. However, it requires a particular model for each system, and there is no applicable model for ARS. Thus, this paper proposes a new model that focuses on the topological relationships between two routes. In this model, railway tracks are expressed as a digraph in which a node and an edge respectively represent a route and a topological relationship between two routes. By analysing topological aspects of railway tracks, we found that edges are categorised into 23 patterns. Each is associated with a specific kind of routesetting feature. For example, a train has to choose an appropriate route where two routes diverge or wait until higher priority trains pass where two routes confluent. By using this model, the ARS system can be designed as follows. Trains move on the digraph and occupy nodes on their path in order to set routes. Each node and edge in the model is linked to specific route-setting functions. When a train tries to occupy a node on its path, ARS executes the functions linked to the node and its connecting edges to decide whether the train can occupy it or not. Some trial systems which have the same functions as existing ARS systems were developed for the feasibility study and were confirmed to ensure high productivity and customisability of the new ARS. Keywords: Automatic Route Setting, track layout, modelling.
\end{abstract}




\section{Introduction}

As computer technologies have advanced, Traffic Management Systems (TMS) have become used extensively for railway operation management and facility control [1]. In the early use of TMS, signallers manually controlled trackside facilities via a human-machine interface at a proper time, but modern TMS integrates an Automatic Route Setting (ARS) system to set the proper route automatically when a train approaches a signal [3,4]. ARS automatically sets routes in accordance with timetables, train descriptions, and facility situations [5].

To develop ARS systems, various conditions have to be considered, such as train operations, track layouts, and equipped facilities. Conventionally, ARS has been developed by highly skilled engineers who can come up with appropriate functions for individual systems to fit with the signalling scheme plan, interlocking control tables, and timetable. This leads to a huge amount of work for the ARS design, implementation, and testing. Therefore, efficiency must be improved to shorten development time and reduce costs. The overall objective of this paper is to improve productivity of ARS development.

In the software development area, various technologies have been proposed for this purpose. One of the commonly used technologies in recent years has been model-based development [6]. To develop TMS, there have been a number of studies about the interlocking systems. Svendsen et al. [7] proposed a domain specific language which automatically generates interlocking tables and interlocking source code from the models drawn on the graphical editor. This model-based domain specific language is useful to improve the productivity of interlocking systems. However, it is a special model for the interlocking system and cannot be applied for the ARS development. Thus, this paper proposes a new model applicable to the ARS development.

In this paper, section 2 presents the overview of ARS systems. Section 3 describes the modelling viewpoint and derivation of the proposed model. Section 4 explains the working principle of model-based ARS and application for some trial systems. Finally, section 5 concludes the paper.

\section{Overview of ARS}

ARS is a major subsystem of railway TMS. Figure 1 shows the relationships between ARS and other components in TMS. The main roles of the ARS [5] are shown below:

a) Automatic route selection when approaching a facing point signal:

ARS selects and sets proper routes for each train where the rail track diverges or trains can reverse their moving direction. ARS usually selects routes on the basis of the timetable information. However, in some sophisticated systems, ARS automatically diverts trains when some trouble has occurred on their planned running path.

b) Output of the route setting command at a proper time:

ARS sends route setting commands to interlocking systems [2] when a train approaches a signal. Basically, route setting timing is scheduled on the 
timetable, but trains may not actually come to a signal punctually because of the flood of passengers and facility trouble. Sometimes signallers may manually set routes which are not on the scheduled path of the trains. Therefore, ARS has to decide appropriate route setting timings by using real-time information of train descriptions and facility situations.

c) Solving conflicts of conflicting routes and calculating train priority:

In a railway system, trains have to run on a rail track and can pass each other only at a limited number of locations. Thus, ARS has to manage running orders of the trains appropriately at each point on the track to avoid causing train delay and deadlock. These running orders have to be taken care of especially on single track lines or large locations which have complex interlocking arrangements.

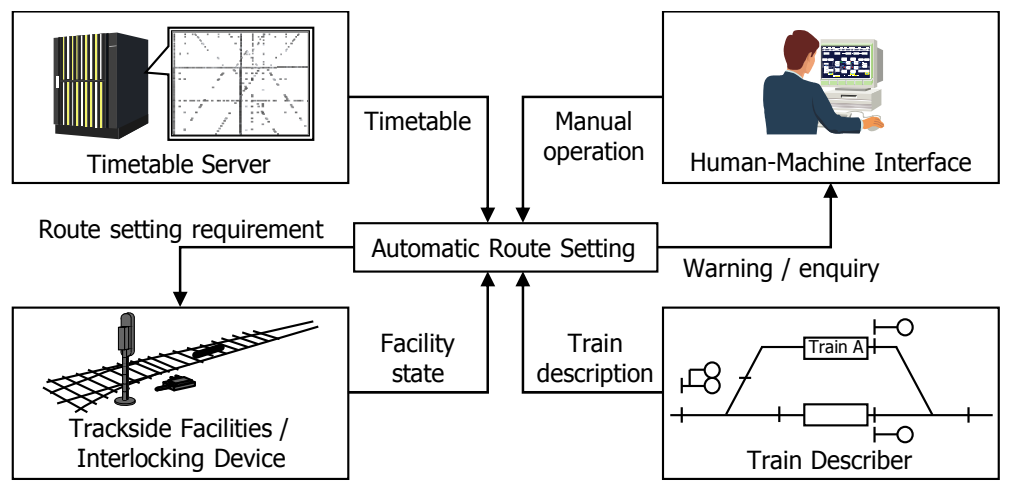

Figure 1: Relationships between ARS and other components in TMS.

\section{Modelling}

\subsection{Modelling of entities on track}

As mentioned before, ARS automatically sets routes for trains running on a track. The "route" is the path along a section of track between one signal and the next, along which authorised movement is to be made. All routes have only one entry signal at their beginning point. Routes also have one exit signal at their destination point, except for the ones whose destinations are bay platforms or dead-end sidings. Train existences and their descriptions are obtained by the train describer system for each "berth", which is a location where a train has to stop when a signal tells it to [8]. Each berth is normally associated with a signal. With routes and berths, rail tracks are modelled as a digraph whose nodes represent berths and whose edges represent routes. This digraph is denoted as the berth-route graph below, an example of which is shown in Figure 2. 


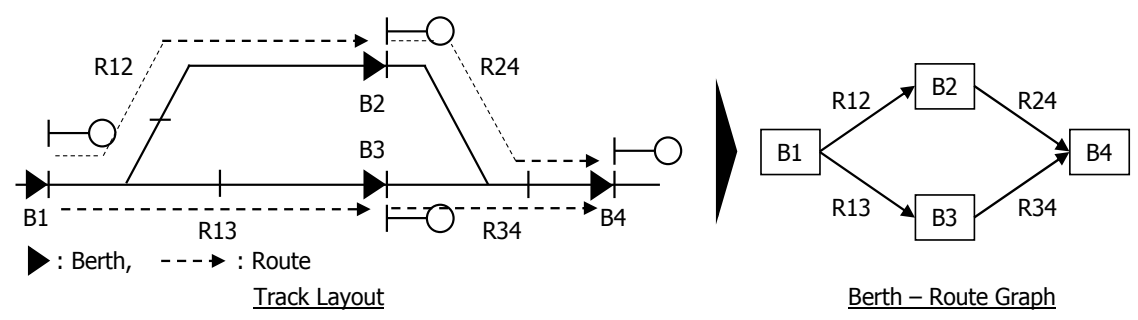

Figure 2: Example of berth-route graph.

In this digraph, train movement is represented as below:

- $\quad$ Each train on the track is assigned on a node

- Trains can step along edges to the edge's direction

- Trains cannot step to the next node without movement authority (i.e. trains can only proceed on routes which have already been set by ARS or signallers)

ARS gives a movement authority for a train by setting a route. When ARS attempts to set a route, ARS has to check the state of conflicting routes. For example, consider a case in which Train A is on Berth B2 shown in Figure 2. ARS will attempt to set the route R24 for Train A in this case, but ARS cannot set it if conflicting Route R34 has already been set. ARS also has to wait to set Route R24 if the train operation plan determines that another train, Train B, has to proceed on Route R34 before Train A proceeds on Route R24. This kind of confliction information among routes cannot be obtained from the berth-route graph. Therefore, ARS needs another model which can describe relationships between two routes. We focus on the geometries of the track layout and model topological relationships between two routes in the next section.

\subsection{Modelling of relationships between two routes}

Appropriate classification criteria have to be chosen to make a well-suited model. We focus on the base points of a route (i.e. entry and exit points of a route) and decide the classification criteria as below:

- $\quad$ Base point overlapping between two routes (section 3.2.1)

- $\quad$ Base point inclusion in the other routes (section 3.2.2)

- $\quad$ Relative direction between two routes (section 3.2.3)

In this section, we explain the reasons we focused on the criteria above and then give details of the model and a list of model elements.

\subsubsection{Base point overlapping between two routes}

The base points of a route have special properties which no other points in a route have. Under the operation of ARS, trains only stop at the base points of a route because train drivers can only obtain the information of whether the train should proceed or not from the signal aspect. It is also special that a train can choose its running path only at the base points because of the interlocking 
mechanism. When a route is set, all points included in the route are locked by the interlocking system. The interlocking system will continue to lock them until the train reaches the exit point of the route. Thus, after the entrance of a route, the train cannot change its running path in the middle of the route even if there is a branch in the interior of the route.

The below characteristics show that ARS functions are affected whether or not the base points between two routes overlap:

- ARS is only able to select train paths where more than two routes overlap at their entry points.

- A train running on a route can only proceed to the next route whose entry point overlaps with the exit point of the route on which train is running.

- $\quad$ Two train paths will never be confluent except for the points where exit points of the routes included in each train path overlap. If a train has been diverted from its running path by the ARS, it can only return to the original running path at the points above.

Figure 3 shows some examples of the base point overlapping between two routes. This figure shows the overlapping of (a) entry points between two routes, (b) exit points between two routes, and (c) exit point and entry point of each route.

(a)

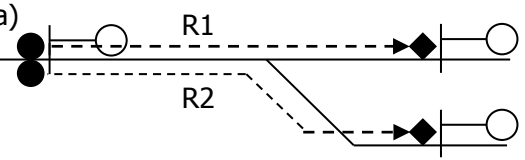

(b)

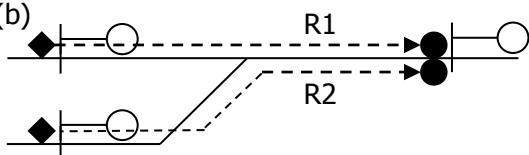

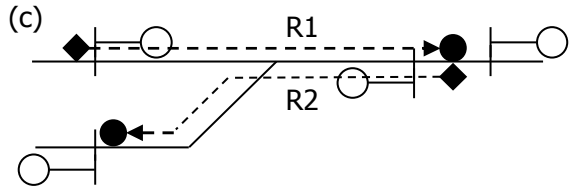

: Entry point of a route

: Exit point of a route $\longmapsto$ : Main signal

Figure 3: Base point overlapping between two routes.

\subsubsection{Base point inclusion in the other route}

Even if the base points of the two routes do not overlap, ARS functions are affected whether or not the base point of a route is included in another route as below:

- If two routes have an intersection point but do not include any of the other's entry points, ARS has to determine the route setting priority among the trains which will enter these two routes. In contrast, there is no degree of freedom of the route setting priority between two routes of which at least one entry point is included in the other one.

- If the exit point of a route is included in the other one, the latter route may not be set for long because a train running on the former 
route may stop at the exit point of the route. Trains that needs to reverse, split, or join at the exit point of the former route may significantly delay other trains.

Figure 4 shows some examples of the base point inclusion between two routes. The figure shows examples in which (a) two routes share no base point, (b) the exit point of one route is included in the other, and (c) the entry point of one route is included in the other.

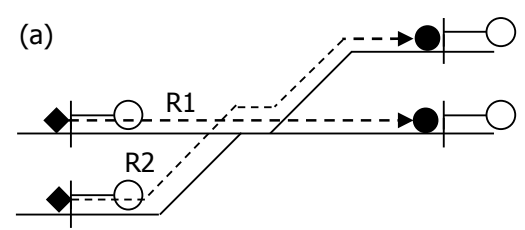

(b)

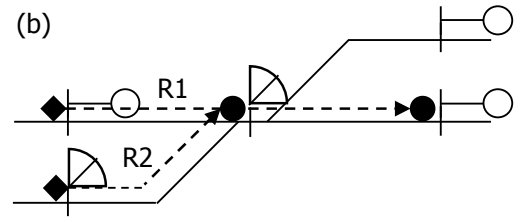

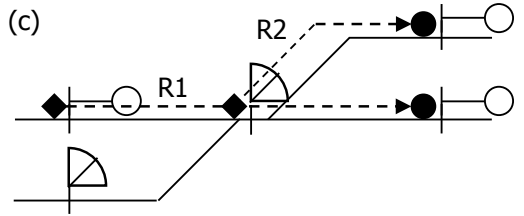

$\checkmark$ : Entry point of a route

: Exit point of a route

$\longmapsto$ : Main signal

$\triangle \quad$ : Shunt signal

Figure 4: Base point inclusion in the other route.

\subsubsection{Relative direction between two routes}

Normally, curvature of a track is sufficiently smaller than the length of a route. Therefore, relative direction between two routes is expressed as two patterns: the same direction or opposite directions. Since a train can move only on a track in railway systems, trains have to switch their head when they intend to go in the reverse direction. Figure 5 shows some examples of relative directions between two routes. While exit point of a route is overlapped with the entry point of the other route in both examples, relative directions between two routes are different. Two routes have the same relative direction in (a) but opposite directions in (b).

(a)

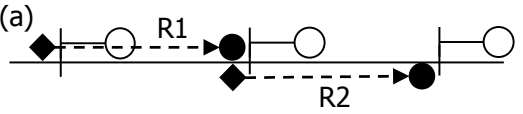

: Entry point of a route

: Exit point of a route

$\vdash$ : Main signal (b)

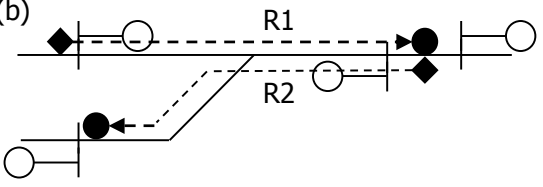

Figure 5: Relative direction between two routes. 


\subsubsection{Topological Route Relation Model (TRRM)}

On the basis of considerations from sections 3.2.1 to 3.2.3, we modelled the topological relationships between two routes as Topological Route Relation Model (TRRM). Table 1 lists all elements in TRRM.

In Table 1, two routes which have a relationship are denoted as Route A and Route $\mathrm{B}$, respectively. We considered that the patterns which coincide by exchanging Route A and Route B are the same pattern. Table 1 is devised to be made so that the same patterns do not appear twice or more with the following rules.

- Only show the cases in which the "Number of base points of Route B included in Route A" is the same as or more than the "Number of base points of Route A included in Route B".

- If the number shown above is the same, only show the cases concerning the entry point of Route B and exit point of Route A.

TRRM assumes that curvature of a track is sufficiently smaller than the length of a route. Patterns which cannot be realised physically under this assumption are denoted as "Unrealisable". Table 1 covers all the patterns obtained by the criteria above.

As shown in Table 1, the elements in TRRM are categorised into 23 patterns. In the figures shown above, Figure 3 (a), (b), and (c) show Patterns 16, 13, and 19, respectively. Figure 4 (a), (b), and (c) show Patterns 1, 5, 3, respectively. Figure 5 (a) and (b) show Patterns 12 and 13, respectively. With the TRRM, rail tracks are modelled as a digraph whose nodes represent routes and whose edges represent the elements of TRRM. This digraph is denoted as the route-topology graph, an example of which is shown in Figure 6.

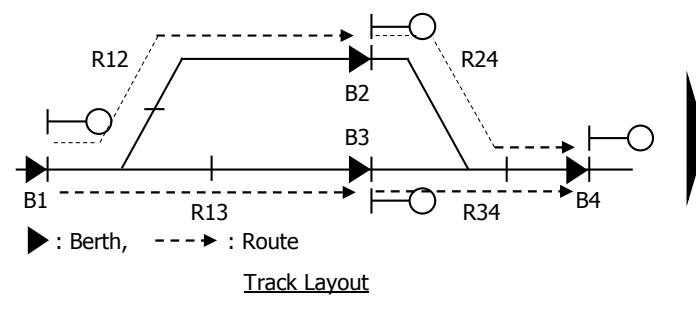

Figure 6: Route-topology graph.

Each element of TRRM is associated with the specific ARS functions as a route selection function or a priority calculation function. Figure 7 shows an example of the association between some elements of TRRM and ARS functions. 
Table 1: Classification of topological patterns between two routes.

\begin{tabular}{|c|c|c|c|c|c|c|c|c|}
\hline & & & & Criteria & & & & \\
\hline No. & $\begin{array}{c}\text { Number of } \\
\text { overlapping } \\
\text { base points }\end{array}$ & $\begin{array}{c}\text { Kind of } \\
\text { overlapping } \\
\text { base points }\end{array}$ & \begin{tabular}{|c|} 
Number of \\
base points \\
of Route B \\
included in \\
Route A
\end{tabular} & $\begin{array}{c}\text { Kind of } \\
\text { base points } \\
\text { of Route B } \\
\text { included in } \\
\text { Route A }\end{array}$ & $\begin{array}{c}\text { Number of } \\
\text { base points } \\
\text { of Route A } \\
\text { included in } \\
\text { Route B }\end{array}$ & \begin{tabular}{|c|} 
Kind of \\
base points \\
of Route A \\
included in \\
Route B
\end{tabular} & $\begin{array}{c}\text { Relative } \\
\text { direction } \\
\text { between } \\
\text { two routes }\end{array}$ & Pattern identity \\
\hline 1 & & & & & & & Same & (1) Simple crossing A \\
\hline 2 & & & & & & & Opposite & (2) Simple crossing B \\
\hline 3 & & & & Entru nint & & None & Same & (3) Entry point blockage A \\
\hline 4 & & & 1 & & 0 & & Opposite & (4) Entry point blockage B \\
\hline 5 & & & & Fyit noint & & None & Same & (5) Exit point blockage $\mathrm{A}$ \\
\hline 6 & & & & & & & Opposite & (6) Exit point blockage B \\
\hline 7 & 0 & None & & Entry noint & & Fxit noint & Same & (7) Entry-exit alternate point blockage \\
\hline 8 & & & & & & & Opposite & Unrealisable \\
\hline 9 & & & 1 & Fntry noint & 1 & Entrunoint & Same & Unrealisable \\
\hline 10 & & & & & & & Opposite & (8) Deadlock B \\
\hline 11 & & & & & & & Same & Unrealisable \\
\hline 12 & & & & & & & Opposite & (9) Both exit point blockage $\mathrm{C}$ \\
\hline 13 & & & 2 & Roth & 0 & None & Same & (10) Entry-exit point blockage A \\
\hline 14 & & & & & & & Opposite & (11) Entry-exit point blockage B \\
\hline 15 & \multirow{14}{*}{1} & \multirow{6}{*}{$\begin{array}{c}\text { Entry point } \\
\text { ofRoute B } \\
\text { and } \\
\text { exit point } \\
\text { of Route A }\end{array}$} & \multirow{2}{*}{0} & \multirow{2}{*}{ None } & \multirow{2}{*}{0} & \multirow{2}{*}{ None } & Same & (12) Forward direction connection \\
\hline 16 & & & & & & & Opposite & (13) Reverse direction connection A \\
\hline 17 & & & \multirow{4}{*}{1} & \multirow{2}{*}{ Entry point } & \multirow{4}{*}{0} & \multirow{2}{*}{ None } & Same & Unrealisable \\
\hline 18 & & & & & & & Opposite & (14) Reverse direction connection B \\
\hline 19 & & & & \multirow{2}{*}{ Exit point } & & \multirow{2}{*}{ None } & Same & Unrealisable \\
\hline 20 & & & & & & & Opposite & (15) Reverse direction connection C \\
\hline 21 & & \multirow{4}{*}{$\begin{array}{c}\text { Both entry } \\
\text { points }\end{array}$} & \multirow{2}{*}{0} & \multirow{2}{*}{ None } & \multirow{2}{*}{0} & \multirow{2}{*}{ None } & Same & (16) Route selection A \\
\hline 22 & & & & & & & Opposite & (17) Route selection B \\
\hline 23 & & & \multirow{2}{*}{1} & \multirow{2}{*}{ Exit point } & \multirow{2}{*}{0} & \multirow{2}{*}{ None } & Same & (18) Route selection C \\
\hline 24 & & & & & & & Opposite & Unrealisable \\
\hline 25 & & \multirow{4}{*}{$\begin{array}{l}\text { Both exit } \\
\text { points }\end{array}$} & \multirow{2}{*}{0} & \multirow{2}{*}{ None } & \multirow{2}{*}{0} & \multirow{2}{*}{ None } & Same & (19) Both exit point blockage A \\
\hline 26 & & & & & & & Opposite & (20) Both exit point blockage B \\
\hline 27 & & & 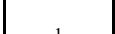 & F & 0 & Vone & Same & (21) Entry-exit point blockage C \\
\hline 28 & & & & & & & Opposite & Unrealisable \\
\hline 29 & \multirow{4}{*}{2} & \multirow{2}{*}{$\begin{array}{c}\text { Each entry- } \\
\text { exit points }\end{array}$} & 0 & Nort & 0 & Non & Same & Unrealisable \\
\hline 30 & & & & & & & Opposite & (22) Deadlock A \\
\hline 31 & & Entry-entry & 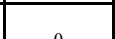 & Non & 0 & Nom & Same & (23) Same base point \\
\hline 32 & & & & & & & Opposite & Unrealisable \\
\hline
\end{tabular}




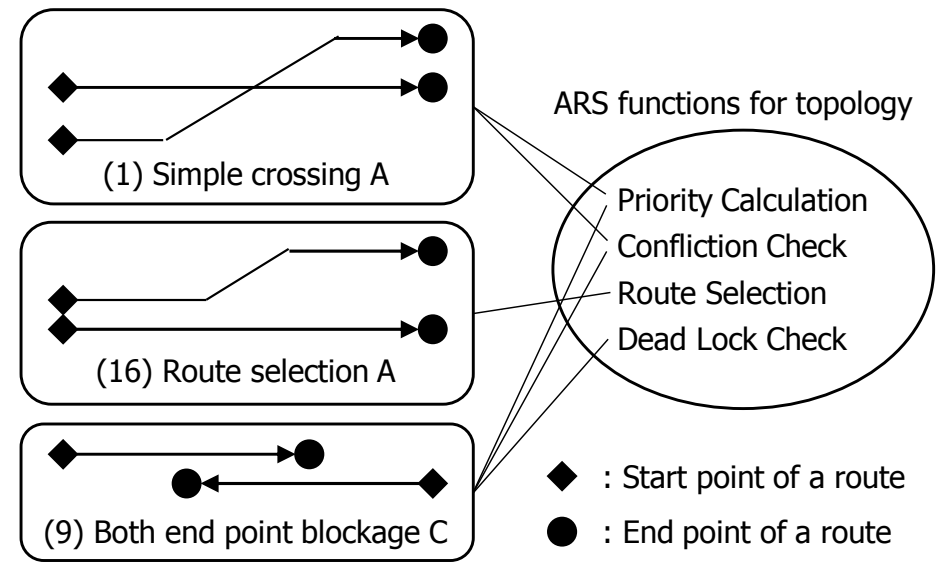

Figure 7: Association between ARS functions and TRRM elements.

\subsection{Model evaluation}

To evaluate the models described in section 3.1 and 3.2, we picked up some sample areas and applied these models to it. Here, any track layouts can clearly be expressed as berth-route graph by the definition of berth and route. Table 2 lists the results of the application of TRRM to these sample areas.

All areas listed in Table 2 can be modelled by TRRM without any exception. The number of TRRM elements in an area is expected to correlate closely with software development effort of ARS for the area because each element in TRRM is associated with specific ARS functions as shown in Figure 7. Comparing Area B with Area A in Table 2, while Area B has fewer routes than Area A, it contains many more TRRM elements. This implies that the track layout of Area B is more complex than that of Area A and that developing an ARS system for Area B needs much effort. Area $\mathrm{C}$ has an astonishing number of TRRM elements, so an ARS system for this area would be extraordinarily difficult to develop. To focus on the patterns which do not have the base point overlapping (i.e. patterns 1-11), while Area A has many elements of Patterns 1 and 2; Area B has many elements of Patterns 3 to 6. This implies that trains simply cross each other in Area A but they can stop or turn back in the crossing point in Area B. Thus, Area B may have more risks of train delay propagation and deadlocking.

\section{Model application to ARS}

This section presents the results of applying TRRM to some trial ARS systems. As mentioned above, each element in TRRM is associated with the specific ARS functions. Figure 8 shows the basic working principle of the ARS based on TRRM. 
Table 2: Application results of the Topological Route Relation Model.

\begin{tabular}{|c|c|c|c|c|}
\hline & Area A & Area B & Area C \\
\hline \multicolumn{2}{|c|}{ Route } & 277 & 225 & 736 \\
\hline \multirow{24}{*}{ 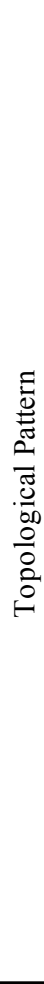 } & 1 & 60 & 7 & 3178 \\
\hline & 2 & 70 & 12 & 1918 \\
\hline & 3 & 0 & 88 & 128 \\
\hline & 4 & 0 & 68 & 687 \\
\hline & 5 & 4 & 33 & 136 \\
\hline & 6 & 34 & 48 & 1979 \\
\hline & 7 & 0 & 0 & 0 \\
\hline & 8 & 0 & 0 & 66 \\
\hline & 9 & 0 & 0 & 218 \\
\hline & 10 & 0 & 2 & 0 \\
\hline & 11 & 0 & 1 & 145 \\
\hline & 12 & 202 & 284 & 2415 \\
\hline & 13 & 102 & 119 & 636 \\
\hline & 14 & 0 & 42 & 49 \\
\hline & 15 & 11 & 18 & 157 \\
\hline & 16 & 81 & 100 & 2642 \\
\hline & 17 & 27 & 57 & 199 \\
\hline & 18 & 4 & 16 & 30 \\
\hline & 19 & 92 & 40 & 1567 \\
\hline & 20 & 25 & 62 & 393 \\
\hline & 21 & 0 & 42 & 47 \\
\hline & 22 & 15 & 66 & 69 \\
\hline & 23 & 0 & 23 & 327 \\
\hline & Total & 727 & 1128 & 16986 \\
\hline
\end{tabular}

As shown in Figure 8, trains running on a track are put into the berth-route graph and step to the adjacent berths on the graph. The ARS attempts to set routes on a train path from the near side of the train until the ARS encounters a route which cannot be set now. When the ARS attempts to set a route, the ARS looks for the route from the route-topology graph and obtains all the TRRM elements associated with the target route. After that, the ARS calls all the check functions associated with the target route and the obtained TRRM elements. Each check function returns whether the route can be set or not, and the ARS sets the target route only if all the check functions allow it to be set. Each route set by the ARS is occupied by the train which has the route on its running path. Trains are only able to proceed on the routes which are occupied by them.

Some trial systems were developed for the abovementioned feasibility study of the TRRM-based ARS. These trial systems were for Areas A and B in Table 2 and had the same functions as existing ARS systems. The partial track layout of the trial areas is shown in Figure 9. 
i) Train tries to set routes on its path from the front side.

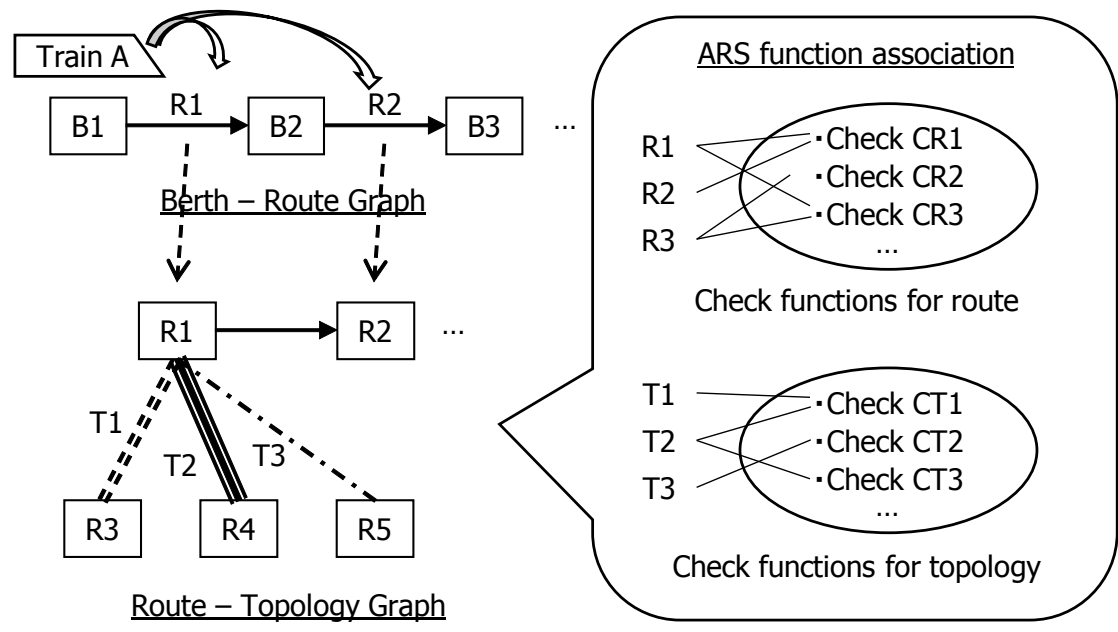

ii) ARS executes all check functions associated with and topologies connected to the target route.

Figure 8: Overview of the ARS working principle based on TRRM.

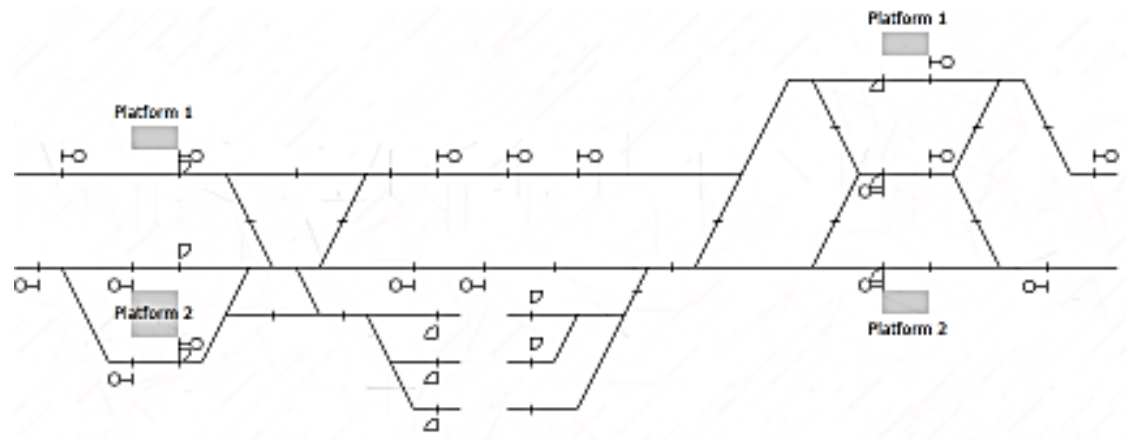

Figure 9: Partial track layout of sample area.

The trial systems were implemented in $\mathrm{C}++$ and worked on the Linux-based real-time operating system. With the trial systems, we confirmed that our new ARS method based on TRRM worked sufficiently well. 
By using TRRM, individual ARS systems can be developed easily from their track layout. Engineers convert a track layout into berth-route graph and route-topology graph, and then all ARS functions can be automatically determined by the elements in the route-topology graph. This implies that TRRM significantly improves the ARS productivity and provides an easy way to fit the individual ARS products to their own track layout.

\section{Conclusion}

This paper presents the new model named TRRM to improve the productivity of ARS systems. TRRM is the model of topological relationships between two routes. TRRM has 23 elements and any tracks can be expressed by them. The basic principles of the new ARS system based on TRRM were explained in this paper, and some trial systems were developed for a feasibility study of it. With the trial systems, we confirmed that our new ARS system based on TRRM worked sufficiently well. Now we are developing some other trial systems for the feasibility study of more complex areas. We are also developing an ARS development system based on TRRM for a much more productive ARS.

\section{References}

[1] M. Harada et al., Shinkansen Traffic Management System for Mutual Direct Operation of Sanyo Shinkansen and Kyushu Shinkansen. Hitachi Review, 61(7), pp. 331-335, 2012.

[2] A. H. Cribbens, Solid-state interlocking (SSI): an integrated electronic signalling system for mainline railways, IEE Proceedings B (Electric Power Applications), 134(3), pp. 148-158, 1987.

[3] M. Kuhn, Automatic route setting integrated in a modern traffic management system. Developments in Mass Transit Systems, 453, pp. 140-145, 1998.

[4] S. Sharples, The Impact of Automation in Rail Signalling Operations. Proc. of the Institution of Mechanical Engineers, Part F: Journal of Rail and Rapid Transit, 225(2), pp. 179-191, 2011.

[5] J. Pachl, Railway Operation and Control, VTD Rail Publishing: 2009.

[6] S. D. Eppinger et al., A model-based method for organizing tasks in product development, Research in Engineering Design, 6(1), pp. 1-13, 1994.

[7] A. Svendsen et al., Formalizing Train Control Language: Automating Analysis of Train Stations. Proc. of the 12th Int. Conf. on Computer System Design and Operation in Railways and Other Transit Systems, pp. 245-256, 2010.

[8] L. J. Bental et al., A Computer Controlled Train Describer, Radio and Electronic Engineer, 38(6), pp. 361-368, 1969. 